\title{
El pensamiento de Lévinas, una lectura fenomenológica y estética.
}

\author{
The thought of Lévinas, a phenomenological and aesthetic \\ reading.
}

Esta obra está bajo una Licencia Creative Commons Atribución 4.0 Internacional. DOI: $10.32870 /$ sincronia.axxii.n74.7b18

\author{
Juan García Hernández \\ Benemérita Universidad Autónoma de Puebla \\ juan.gh.gh@hotmail.com \\ (MÉXICO)
}

Recibido: $31 / 03 / 2018$

Revisado: 20/04/2018

Aprobado: $12 / 06 / 2018$

\section{RESUMEN}

El presente trabajo tiene como objetivo primordial reflexionar alrededor del pensamiento de Emmanuel Lévinas a la luz de una breve revisión de aspectos centrales de su proyecto filosófico, tales como realidad, tiempo, sombra y entretiempo con la intención de mostrar rasgos característicos de la propuesta fenomenológica del filósofo judío, en contraste con sus ideas estéticas. Mediante la exposición intentaremos obtener resultados parciales que nos arrojen satisfactoriamente a una tematización válida, cabe destacar que la intención de rehabilitar el diálogo entre la fenomenología de Lévinas y su análisis alrededor de problemas estéticos se ajusta a un proyecto de investigación que pretende redescubrir un texto que ha sido paulatinamente invisibilizado como La Realidad y su sombra, aunado a tal hecho la exposición busca conjuntar un aparato crítico de conceptos que pueda brindar herramientas para una mejor lectura alrededor del pensamiento levinasiano.

Palabras clave: Arte. Verdad. Sensibilidad. Tiempo. Realidad. Sombra.

\section{ABSTRACT}

The main objective of this paper is to reflect on the thinking of Emmanuel Lévinas in the light of a brief review of central aspects of his philosophical project, such as reality, time, shadow and halftime with the intention of showing characteristic features of the phenomenological proposal of the Jewish philosopher, in contrast to his aesthetic ideas. Through the exhibition we will try to obtain partial results that satisfactorily throw us into a valid theming, it should be noted that the intention to 
rehabilitate the dialogue between the phenomenology of Lévinas and its analysis around aesthetic problems fits a research project that aims to rediscover a text that has been gradually invisible as The Reality and its shadow, coupled with this fact the exhibition seeks to bring together a critical apparatus of concepts that can provide tools for a better reading around Levinasian thought.

Keywords: Art. Truth. Sensitivity. Time. Reality. Shadow.

\section{Desarrollo}

La obra de Emmanuel Lévinas se extiende a lo largo de 60 años desde sus primeras publicaciones en la década de 1930 hasta sus últimos trabajos cercanos a 1980 este hecho nos muestra que el acercamiento a la prolífica obra del pensador judío exige una atención delicada y sutil, ya que de fondo existe un largo recorrido que el filósofo nacido en Lituania ha de presuponer entorno a su propia obra, es decir que conforme avancemos en la lectura de su trabajo habrá conceptos, esquemas y relaciones que podríamos pasar por alto no si antes desenmarañamos las problemáticas centrales que pretende dilucidar, teniendo en cuenta este hecho la presente exposición tiene como principal objetivo identificar algunos aspectos centrales de la obra levinasiana para aproximarse en un sentido muy general a la filosofía del pensador judío, para ello también nos serviremos de algunas ideas sobre arte y poesía que el filósofo en cuestión publicó con la intención de rehabilitar la reflexión en torno al fenómeno artístico en vistas de establecer un puente entre aquello que para varios críticos del pensador lituano queda al margen favoreciendo la centralización de su obra en una raíz puramente fenomenológica-ética sin notar que pueden atisbarse ciertos contrastes entre dicha raíz y el fenómeno artístico, estos contrastes pueden alumbrar la rehabilitación de ciertos problemas que valen la pena seguir poniendo sobre la mesa.

Una vez planteado el objetivo general de esta exposición, hemos de tratar los caminos específicos sobre los cuales se desplegará, para ello resulta necesario dividir el trabajo en al menos tres ejes, dichos ejes se dividirán en primera instancia a partir de dos problemas que logramos identificar, tales problemas se presentan a partir de los conceptos de realidad y tiempo tales conceptos derivarán subtemas entre líneas bajo la pretensión de tratar el contraste que arriba 
anunciamos, que a su vez brotarán nociones como sombra y entretiempo. Notificada la vía que transitaremos esperamos hallar resultados satisfactorios al final de nuestra exposición.

El trabajo de Lévinas se consolidó rápidamente en el circuito de la filosofía académica francesa, al arribar a Francia marcado por circunstancias políticas de su país natal y motivado por un ímpetu político que más tarde se verá reflejado en sus postulados éticos, no sin antes transitar un recorrido sobre la filosofía alemana específicamente de la filosofía que se trabajaba en Friburgo a finales de los años 20’s y principios de los 30’s, dicha vinculación lo llevó a trabajar un método que será fundamental para el desarrollo de su propio pensamiento: a saber, el método fenomenológico, a raíz de la vinculación que estableció con Husserl y Heidegger, hicieron que su paso por Friburgo aquella ciudad "pequeña, pulcra y alegre que acoge un Romanticismo salvaje y una catedral que es 'rosa como una prometida'” (Lévinas, 2006, p. 89), fuera sumamente productivo ya que en su regreso a Francia con la publicación de la obra La teoría de la intuición en la fenomenología de Husserl que a la postre servirá como tesis de doctorado y por ende lo catapultará de joven prospecto a pensador eminente de la filosofía francesa contemporánea al ser de los primeros en recibir y divulgar la propuesta fenomenológica con un trabajo que después cobrará frutos al convertirse en una lectura obligada para las futuras generaciones francesas, generaciones notables como la del mismo Jean Paul Sartre.

A grandes rasgos podemos recuperar algunas ideas generales alrededor de la obra que permitió a Lévinas ascender de grado académico, en la medida en que se ponen al menos dos ideas en juego, la idea de lo sentido como mediación y la idea de intuición no como modo de conocimiento sino como el fenómeno primitivo que hace posible la verdad misma, ideas que serán las impulsoras para que el filósofo lituano trabajé varios años a partir del método fenomenológico que por cierto lo define en dicha tesis doctoral como "una manera de filosofar que no se agota, no como un instrumento para explicar lo real sino como un proceder que tiene una visión anticipada del sentido del ser que se aborda" (Lévinas, 2004, pág. 21). Ya desde aquí, puede notarse una mirada aguda al identificar justamente que el problema central no viene dado por parámetros 
epistemológicos sino más bien ontológicos, diferencia que yace presente con la primera idea que gira alrededor de lo sentido como mediación y no como absoluta o trascendental, es decir que una sensación no viene dada por un grado de representación que de acuerdo a Kant sí ocurre justamente porque para el filósofo de Königsberg el sujeto dotado de sensibilidad asimila el mundo a partir de condiciones a priori absolutas que se opone a las condiciones a posteriori, originadas en la experiencia o en palabras suyas “¿cómo podría ser despertada la facultad de conocer sino mediante objetos que afectan nuestros sentidos y que ora producen representaciones? [...] entendiendo como conocimiento a priori el que es absolutamente independiente de la experiencia" (Kant, 2010, pp. 39-41).

Si bien, la anterior cita a expensas de una vista microscópica nos permite corroborar que efectivamente para Kant podemos asimilar el mundo a partir de sensaciones puras, premisa que terminará criticando Husserl ya que para él, más bien se debe hablar de sensaciones mediadas, esto quiere decir que asimilamos y captamos al mundo interpretando, esta tesis se ve reforzada gracias al pasar de los estratos biológicos hacia los estratos del sujeto donde se da una mediación subjetiva, lo cual es relativo al sujeto esto ya se opone radicalmente frente al naturalismo, el cual construye su objeto a través de un supuesto universal, y efectivamente la fenomenología no se basa en supuestos universales y por ello el proceso de mediación puede definirse como aquel proceso del cual obtenemos un conocimiento no-universal, de éste concluye el conocimiento de algo singular lo que nos da para pensar por ejemplo en la esencia de la computadora, esto puede clarificarse con el siguiente párrafo:

para el naturalismo la objetividad misma de un fenómeno físico implica la existencia del mundo físico, ser para él no significa necesariamente tener una existencia material pero si encontrarse ahí del mismo modo en que el mundo material lo está, ser en el mismo plano que él. Pensar algo como existente es pensar en algo en la naturaleza física. La objetividad, la realidad, la existencia del fenómeno desaparecerían si lo despojáramos de su pertenencia a la naturaleza (Lévinas, 2004, prólogo) 
La anterior cita clarifica de mejor modo lo que hasta ahora hemos planteado es decir que para Lévinas y por ende para Husserl. La fenomenología, puede dar la posibilidad de asimilar el mundo a partir de mediaciones y a su vez, éstas hacen posible la pregunta por las esencias. Este modo de proceder implicará un límite, el cual es dado como un factor importantísimo para asimilar el mundo y en suma la realidad, ya que el camino esbozado por la fenomenología tiene por ruta principal la pregunta por los modos en que se nos aparecen las cosas, este hecho deja a un lado la similitud que podría describirnos frente a los animales, a ellos no se les aparecen las cosas, pero a nosotros sí, porque tenemos la capacidad de distinguir realidades y esto a través de mediaciones, en otras palabras podemos decir que hay una esfera que rodeamos y al momento de girar vemos, ahí al ver, hay una mediación, y nace una suspensión aún más enigmática; la inteligibilidad. Esto cobra validez para Lévinas no sólo por la propuesta de los pensadores de Friburgo, sino de su propia tradición francesa, donde a partir de la absorción del pensamiento bergsoniano el cual pretende superar el empirismo pensando el ser a partir desde la temporalidad en contraposición de lo estático con lo dinámico, abriendo la posibilidad para cierto ámbito ético, que a la postre será el hilo que permita hacer crecer su filosofía que deviene a partir de conceptos fundamentales como el tiempo.

Hasta ahora podemos decir que la fenomenología para Lévinas fue el parteaguas para dinamizar su recepción de la tradición francesa, por ello no es en vano que, así como lo fue para Heidegger, el problema que ve de fondo es una propuesta ontológica.

Pasando a la segunda idea, sobre la intuición, debemos tener en cuenta que existe un gran abanico de conceptos que en cierta medida exigen un mayor desarrollo si se pretende ser lo suficientemente claro, sin embargo debido a la intención de nuestro trabajo hemos decidido dejar esta idea suspendida y aclararla someramente, a raíz de esto Lévinas realiza la diferencia de dos aspectos fundamentales para la fenomenología, tal diferencia se da entre los actos significativos y los actos intuitivos, los segundos son aquellos en donde frente al objeto, no sólo se piensa sino que se ve, se clarifica con evidencia o en otras palabras "el caso privilegiado de la intuición es la intuición originaria o percepción que se caracteriza no solo por verlo 'en original'” (Lévinas, 2006, p. 80) 
frente a ello hemos de reconocer que ya se empieza a esclarecer cierta problemática que yace de fondo, ni más ni menos que la misma realidad, es decir que en la medida en que esbozamos cierta relación con el objeto y a su vez podemos llegar a definirlo, se establecen ciertos parámetros para distinguir una determinada verdad, sin embargo no puede quedarse ahí dicho análisis y como bien advierte el propio filósofo judío:

[...] la realidad no se resuelve con indicaciones generales ya que para cada categoría de objetos se plantea la cuestión de la realidad y para ver qué significa verdad en cada región hace falta a la conciencia trascendental a fin de mostrar la estructura de los actos intuitivos (Lévinas, 2006, pág. 81).

Cabe destacar que en relación a la verdad tanto para Heidegger como para Lévinas, la cuestión central gira en torno al Ser.

\section{Realidad}

Con lo expuesto arriba podemos comenzar el desarrollo de nuestro trabajo partiendo de una pregunta fundamental tanto para la fenomenología como para el propio filósofo lituano, ¿qué significa estudiar la realidad?, podemos decir que puede fundamentarse como un intento por describir las cosas mismas, sin embargo debemos matizar algunos aspectos entorno a dicha tesis ya que para la corriente fenomenológica la realidad se da en un para alguien, por ende está en juego cierta noción de conciencia, para ello debemos tener en cuenta que en la medida en que nos relacionamos con el mundo y por ende con los objetos, el mundo se nos ofrece como objeto de la conciencia, pero el mundo no se da por si mismo, en carácter objetual de esto resulta que mi mundo siempre es percibido de ahí los conceptos de percepción que inaugura Husserl a lo largo de su obra, de esta cuestión Lévinas propone a la par de la realidad la cual emerge como un supuesto natural cuyo fundamento y objetivo se centra en determinada verdad gnoseológica, propone como antítesis, el concepto de sombra que "se refiere a una imagen de lo real ya exiliado de ella" (Dominguez, 1997, p. 70). 
Para entender esta idea con mayor claridad, debemos asimilar que a mediados de 1948, el filósofo judío publicó un texto alrededor del arte, y las posibilidades que pueden nacer a partir de su análisis y muy específicamente sobre la crítica son múltiples y es justamente a partir de tal ensayo donde podemos atisbar tanto el contraste que establece con la fenomenología ubicándola a partir de un marco de posibilidad racional y por ende conceptual en oposición con el arte, definiéndose como aquello que no se comprende, como anunciando una no-verdad.

En palabras del propio Lévinas “Donde el lenguaje común abdica, el poema y el lienzo hablan. Más real que la realidad, la obra da testimonio de la imaginación artística que se erige en saber de lo absoluto" (Lévinas, 1948, p. 117). He aquí los primeros antagonismos de la definición de realidad que anteriormente al pensamiento levinasiano se propone, es decir que para el filósofo judío la realidad no se presenta como univoca y lineal sino dual en la medida en que la obra artística funge como un cierto anverso de la realidad, es decir la obra artística funge como sombra de lo que es luz, luz en tanto verdad, y sombra como no-verdad, inclusive a riesgo de caer en equívocos podemos atrevernos a decir que para Lévinas la posibilidad de la filosofía se de en ese lado de la verdad, y el arte se da en una dimensión donde no le pertenece aquello que puede decirse, no es vano que para Lévinas la literatura y en general el arte expresen lo inefable y que justo en la medida en que los "críticos de arte" intentan comprender y develar lo dicho por la obra su discurso la vuelve banal y a su vez vulgar, porque parten de categorías pretenciosamente racionales teniendo en cuenta este hecho y en consonancia con algunos escritores como Blanchot, el filósofo nacido en Kaunas se pregunta en relación a este hecho:

en qué consiste la no verdad del ser?, ¿ se define con relación a la verdad, como un residuo del comprender?. El arte no conoce un tipo particular de realidad, contrasta tajantemente con el conocimiento. Es el acontecimiento mismo del oscurecimiento, una llegada de la noche, una invasión de la sombra (Lévinas, 1948, p. 120)

Para ampliar el anterior análisis y demostrar el contraste entre filosofía y arte, debemos tomar en cuenta lo que habla respecto a la imagen, la cual adquiere una función precisa; la imagen en el 
proceder del arte consiste en sustituir el objeto con su imagen, es decir que la imagen adquiere un nuevo proceder en relación al objeto que permanece ausente, esto sirve de contraste en la medida en que mantenemos una relación con el objeto, al captarlo, valiéndose de los actos significativos para constatar un objeto en cambio para el escritor judío, en el arte "la imagen neutraliza esta relación real, significa antes que nada una ceguera de cara a los conceptos" (Lévinas, 1948, p. 122) y esta referencia nos da a entender que el arte no posee ese carácter revelador de la verdad, ya que se cimbra sobre una sombra, una irrealidad, inclusive insignificante como ha querido demostrar en otros textos por ejemplo en Sobre Maurice Blanchot plantea lo siguiente: "la obra de arte, en suma el poema se sitúa para Blanchot fuera del reino del Día, ya que la eficacia del arte como ha demostrado es la más insignificante para la Historia" (Lévinas, 2000, p. 32)

Sin embargo cacaso no cabe preguntarnos si para la fenomenología la cuestión del Ser, es la problemática central en dónde queda el Ser respecto a los planteamientos de Lévinas? Bueno, habrá que replantear que ya el mismo Lévinas se lo preguntaba antes de la publicación del texto Realidad y su sombra.

Dichos cuestionamientos como lo refleja algunas líneas de sus Escritos inéditos 1 anuncia "la última consecuencia de mi concepción estética: la metafísica, ¿es a fin de cuentas un arte, el sentido de la existencia es un arte?, ¿la existencia es un arte?" (Lévinas, 2013, p. 75) y en qué medida estas preguntas no resuenan frente a las tesis de un Heidegger en donde no cabe una consideración respecto a la existencia como un arte en la medida en que el arte se constituye a partir de una estructura distinta que la existencia, ya que precisamente en el arte o en la obra hay una contemplación y dicha "contemplación de la obra significa estar dentro de la patencia del ente que acontece en la obra y tal estancia es un saber y tal es lo sereno de la verdad que acontece en la obra" (Heidegger, 1958, p. 90). Es decir, que para el filósofo de Marburgo, la consideración entorno al arte se erige bajo la posibilidad de que hay verdad dentro de la obra, no obstante para Lévinas ocurre lo contrario, esta no-verdad patente en la obra de arte descansa sobre otro fundamento que es la sensación, no en vano al principio hablamos de lo que significa la sensación para la 
fenomenología, dicho aspecto en Lévinas opera de la siguiente forma en cuanto a sus consideraciones estéticas, " la sensación revela algo que le es propio, por esto la obra de arte es un conocimiento de un género particular, ¿̇no es ser?...es despojamiento" (Lévinas, 2013, p. 74).

De lo anterior, podemos derivar que ese despojamiento o inclusive a expensas de errores, nos atrevemos a decir en términos heideggerianos, aquel salir de sí, yace anclado al mismo sujeto, por consiguiente la posibilidad de encontrarse con una verdad o una no-verdad en la obra de arte son posibilidades que guardan cierto paralelismo entre Heidegger y Lévinas sin embargo la diferencia radica en que para este último las tesis de filósofo alemán como "Ser en el mundo" se da a partir de una existencia con conceptos y esta tesis resulta ajena a la sentencia levinasiana de un" Ser entre las cosas" un ser que exige un aquí y un ahora ya que el ser no es solo en sí mismo, sino se escapa, porque en la medida en hay una persona y gestos, miradas, miembros o piel

[...] todo esto es la persona, es la cosa. Hay entonces en esta persona, en esta cosa, una dualidad, una dualidad con su ser. Ella es lo que es y también extranjera a sí misma, y hay una relación entre estos dos momentos. Diremos que la cosa es ella misma y su imagen (Lévinas, 1948, p. 126).

Hemos llegado a un punto álgido de nuestro trabajo, qué entiende el filósofo judío por imagen y cuál es su relación con la obra de arte y en suma por qué contrastar un contenido de carácter ontológico a uno de carácter estético. Pues en el fondo retornamos a lo que en un principio atisbamos y esto tiene que ver con la realidad, no sólo es lo que es sino también su sombra, su imagen, inclusive resulta válido plantear que la imagen adquiere un nuevo sentido, la de alegoría, la imagen es alegoría del ser y esto qué tiene que ver con el arte, pues que "contemplar una imagen es contemplar un cuadro" (Lévinas, 1948, p. 127), así en relación al fenómeno artístico debemos plantear que el arte nos lleva a una dimensión no que traspasa o rebasa la realidad sino más bien, nos remite a un más acá, a un tiempo en donde nos restituimos como seres. 


\section{Tiempo}

El tiempo en relación a lo hasta ahora expuesto guarda vínculos con las últimas líneas del apartado anterior, para Lévinas la cuestión del tiempo se vuelve central no sólo para la fundamentación de su propuesta ontológica sino también estética, en la medida en que en el caso de sus planteamientos estéticos descubrirá un modo en que el tiempo se relaciona con el ser, dicho modo lo denomina entretiempo, y tal entretiempo colisiona con esa dimensión donde el ser es restituido a un más acá, tal efecto será posible solo si de por medio yace la obra artística, sin embargo antes de pasar a la revisión de este asunto, resulta primordial esbozar al menos en términos muy generales, lo que denota el tiempo en la filosofía levinasiana.

“El tiempo es la expresión del hecho de ser o de ser mismo", por lo tanto si entendemos el ser a partir de la dimensión del tiempo, resulta un existir que es temporalizarse y dicha existencia no es una con otra cosas sino desde esa existencia se da el tiempo ya que no se puede pensar el Dasein como cosa sino como existencia a partir de un tiempo, nada ajeno a los postulados de Ser $y$ tiempo, sin embargo, cabe destacar que la temporalidad del tiempo sólo se entiende en relación a la muerte además ha de considerarse como la posibilidad de posibilidades y en la medida en que se ven inmiscuidos los temples de ánimo a partir de una dimensión de lo afectivo a nivel ontológico, se va articulando tiempo, y en suma épocas originando una manera en que el Dasein configura su temple, por ende no resulta gratuito que se hable de un temple epocal.

Lo anterior implicó para Lévinas la consideración del tiempo como eje fundamental para alguna propuesta ontológica, sin embargo al preguntarse por lo que significa el temporalizarse del tiempo, el pensador judío, establece a diferencia de Heidegger que la posibilidad de posibilidades no es la muerte sino el propio nacimiento ya que dicho acontecimiento da paso a un escenario en donde el sujeto tiene que hacerse cargo de sí, es decir que la apropiación del tiempo fenomenológico en relación al ser ha de entenderse como la aparición de un sujeto que se remite a un yo, a un sí mismo, pero no es la mismidad heideggeriana sino más bien en tanto ser es dos; dos 
en el instante este dos es la relación del yo a sí mismo, que se opone a aquello que es sin relación, se opone a su pura verbalidad, transitividad, se opone algo que es no-relación o en otras palabras ser es lo que se opone al sujeto, tal es la primera relación entre sujeto y ser, debe darse una dimensión de alteridad, donde se es avasallado por lo externo, de aquí han de bosquejarse varios conceptos que la filosofía de Lévinas sugiere, conceptos como la desnudez del rostro, el cuerpo encadenado o "ser es el mal".

Sin embargo lo que hasta aquí nos interesa es la posibilidad de conceptualizar la relación sertiempo a partir del instante, valdrá la pena en algún momento esclarecer en qué medida esta concepción del tiempo se vislumbra en otros autores como Leibinz o Kant y preguntarse si acaso la fenomenología ha superado dichas tematizaciones desplazando la problemática al considerar los fenómenos primarios como configuraciones que no nacen a partir del sujeto más bien que aquellos configuran al sujeto, en esa circunstancia se ponen en juego elementos como el tiempo inmanente, la proto impresión y la sensación, no obstante dejaremos dichas reflexiones sobre la mesa, ya que nuestro interés descansa en rescatar que la sensación da lugar a un primer instante, hay una aquí y un ahora y en tal instancia se da la primera fase temporal arribando a un instante presente que en cierta medida arroja una sensación retenida la cual condicionará el instante que sigue, por ende surge una dialéctica de los instantes pero en este punto el filósofo lituano extiende su análisis en relación al tiempo al considerar que el tiempo es exterior a mi instante porque el tiempo solo se da a partir de lo Otro. He aquí la relación Ser-tiempo en Lévinas, el tiempo en el que se produce el Ser y el infinito va más allá de lo posible.

En cambio en el ensayo Realidad y sombra al constituir la imagen como alegoría del ser, y a su vez la obra como aquello donde se instaura un No-ser, habrá que mirar con atención que la tematización alrededor del tiempo no se queda al margen, el más acá que planteamos arriba, se distancia de las consideraciones entorno al instante y al tiempo que definimos en el párrafo anterior, dicho más acá se ubica en un entretiempo tal tiempo si lo podemos ver así se ubica entre aquello que se vierte como un pasado y aquello que colinda con un futuro, con un provenir, pero en 
relación a la obra artística, ésta habita de cierta forma tal instante en la medida en que se congela, en palabras más concretas "la estatua realiza una paradoja de un instante que dura sin porvenir. Su duración no es realmente instantánea tiene a su manera una duración quasi-eterna, en el interior de la obra el instante dura infinitamente" (Lévinas, 1948, p. 132); sin embargo, habrá que preguntarnos hacia donde se despliega la obra para ello el filósofo lituano advierte "el arte es el movimiento de la caída más acá del tiempo, en el destino" (p. 133). Esto abre un horizonte de problematización donde la producción artística se revela en el tiempo y escapa a su vez a consideraciones conceptuales, podemos atrevernos a decir que toda crítica de arte no esclarece lo que en el fondo persiste en la propia obra, puede decirse que el arte se enfrenta a un no poder pasar del tiempo es decir,

[...] el arte cumple precisamente esta a-duración en el intervalo, en esta esfera que el ser tiene el poder de atravesar pero en la que su sombra de inmoviliza, la estatua difiere radicalmente de la eternidad del concepto-se trata aquí del entretiempo, nunca terminado, que dura aún-algo inhumano y monstruoso (Lévinas, 1948, p. 136)

Como hemos intentado exponer a lo largo del trabajo, nuestro principal objetivo fue delimitar y esclarecer ciertos contrastes entre los planteamientos ontológicos de Lévinas y su postura estética de lo anterior podemos concluir que la rehabilitación de dichos contrastes son vitales para acercarse al pensamiento levinasiano en la medida en que hay oscilaciones de conceptos que fácilmente pueden conllevarnos al arribo de otros horizontes posibilitando una mayor comprensión de su obra además el objetivo de mirar la actualidad y vigencia de la propuesta filosófica de Lévinas, nos lleva a detectar escenarios que se oponen a los abundantes comentarios de la obra del filósofo judío en donde se centran en sus trabajos alrededor de la ontología y la ética cimbrando un aparente olvido sobre sus apuntes alrededor de la estética, comentarios que más allá de ayudar a comprender la obra, la fragmenta. Por ende, la tarea de este trabajo permitió al menos en principio el redescubrimiento de no sólo aspectos ontológicos que van a la base del pensamiento de Lévinas, sino también de ampliaciones problemáticas a partir del estudio de la obra artística. 


\section{Referencias:}

Dominguez, A. (1997). Sombra de la realidad. En La llamada exótica. El pensamiento de Emmanuel Lévinas. Madrid: Trotta.

Heidegger, M. (1958). La verdad y el arte. En Arte y poesía. México: FCE.

Kant, I. (2010). Introducción. En Crítica de la razón pura. Madrid: Gredos.

Lévinas, E. (1948). La realidad y su sombra. En Los imprevistos de la historia. Salamanca: Sigueme.

Lévinas, E. (2000). El día y la noche. En Sobre Maurice Blanchot. Madrid: Trotta.

Lévinas, E. (2004). Prólogo. En La teoría fenomenológica de la intuición. Salamanca: Ediciones Sigueme.

Lévinas, E. (2006). Friburgo, Husserl y la Fenomenología. En Los imprevistos de la historia. Salamanca: Ediciones Sígueme.

Lévinas, E. (2006). Husserl, Heidegger y Jean Wahl. En Los imprevistos de la historia. Salamanca: Sigueme.

Lévinas, E. (2013). Cuaderno 5. En Escritos inéditos 1. Madrid: Trotta. 